\title{
Lanthanum (III) nitrate hexahydrate catalyzed one-pot synthesis of 2-arylbenzothiazoles under mild reaction conditions
}

\author{
Kabeer A. Shaikh $\odot^{1 *}$ and Uddhav N. Chaudhar $\odot^{2}$ \\ ${ }^{1}$ P. G. Department of Chemistry, Sir Sayyed College of Art's, Commerce \& Science, Aurangabad- \\ 431001 [M.S.]-India \\ ${ }^{2}$ Department of Chemistry, Kalikadevi Art's, Science \& Commerce College, Shirur (Ka.) Dist. Beed- \\ 413249 [M.S.]-India
}

(Received August 02, 2017; Revised September 13, 2017; Accepted September 28, 2017)

\begin{abstract}
An efficient one pot synthesis of 2-arylbenzothiazole derivatives through condensation of aldehydes and 2-aminothiophenol in the presence of catalytic amount of $\mathrm{La}\left(\mathrm{NO}_{3}\right)_{3} \cdot 6 \mathrm{H}_{2} \mathrm{O}$ under mild reaction conditions was developed. The key advantages of this protocol are short reaction time, high to excellent yields, simple work up, inexpensive catalyst and simple separation of pure product.
\end{abstract}

Keywords: Aromatic aldehyde; 2-aminothiophenol; Lanthanum (III) nitrate hexahydrate. C 2017 ACG Publications. All rights reserved.

\section{Introduction}

Chemistry of heterocyclic compounds is one of the leading research subjects in organic chemistry. Heterocyclic compounds are widely distributed in nature and are essential for life. They play a vital role in metabolism of all living cells. There are vast numbers of pharmacologically active heterocyclic compounds, many of which are in regular clinical use. Nitrogen, sulfur and oxygen containing five membered heterocyclic compounds have occupied enormous significance in the field of drug discovery process. Benzothiazole and its derivatives are often found in heterocyclic compounds, which exhibit a variety of biological activities, such as anti-viral, ${ }^{1}$ anti-bacterial, ${ }^{2}$ antifungal, ${ }^{3}$ anti-microbial, ${ }^{4}$ anti-Parkinson, ${ }^{5}$ anti-cancer ${ }^{6}$ and anti-tumor. ${ }^{7}$ Moreover, they are also used as drugs for treatment of diabetes. ${ }^{8}$ Benzothiazole unit is found in zopolrestat ${ }^{9}$ and riluzole, ${ }^{10}$ which are used to treat diabetes.

Due to their wide range of synthetic, industrial and pharmacological applications, synthesis of substituted benzothiazoles has become a focus of intense research in recent years. Several synthetic methodologies have been developed for the synthesis of 2-substituted benzothiazoles, including

\footnotetext{
* Corresponding author: E-mail: shaikh_kabeerahmed@rediffmail.com Phone: + (91)-240-2311285, Fax: + (91)240-2313876
} 
condensation of 2-aminothiophenol with substituted nitriles, carboxylic acids, aldehydes, acyl chlorides and esters, followed by an oxidation. Different catalysts were reported to be applied for the synthesis of these heterocycles like silica gel, ${ }^{11} \mathrm{HBF}_{4}-\mathrm{SiO}_{2},{ }^{12}$ bakers' yeast, ${ }^{13} \mathrm{CAN},{ }^{14}$ copper-catalyzed Tandem Reaction, ${ }^{15} \mathrm{~K}_{2} \mathrm{~S}_{2} \mathrm{O}_{8}{ }^{16-17}$ SDS,${ }^{18}$ PEG-400, ${ }^{19}$ L-proline, ${ }^{20}$ PTSA,${ }^{21}$ CTAB, ${ }^{22}$ acetic acid,${ }^{23}$ silica sulfuric acid, ${ }^{24}$ PCC/silica, ${ }^{25}$ SBA-Pr- $\mathrm{SO}_{3} \mathrm{H}^{26}{ }^{20}$ sulfamic acid, ${ }^{27}$ vanadium(IV)salen, ${ }^{28}$ $\mathrm{FeCl}_{3} /$ montmorillonite $\mathrm{K}-10,{ }^{29}$ ammonium chloride, ${ }^{30} \mathrm{Sm}(\mathrm{OTf})_{3},{ }^{31-32} \mathrm{BF}_{3} / \mathrm{SiO}_{2}{ }^{33}$ lithium bromide, ${ }^{34}$ $\mathrm{Ag}_{2} \mathrm{CO}_{3} /$ Celite, ${ }^{35} \mathrm{Fe}_{3} \mathrm{O}_{4} @ \mathrm{SiO}_{2} /$ collagen, ${ }^{36}$ ruthenium (I) complexes, ${ }^{37} \mathrm{VOSO}_{4}{ }^{38} \mathrm{Co}\left(\mathrm{NO}_{3}\right)_{2} \quad 6 \mathrm{H}_{2} \mathrm{O},{ }^{39}$ $\mathrm{Fe}\left(\mathrm{NO}_{3}\right)_{3}: 9 \mathrm{H}_{2} \mathrm{O},{ }^{40} \mathrm{Bi}\left(\mathrm{NO}_{3}\right)_{3},{ }^{41} \mathrm{CuSO}_{4},{ }^{42} \mathrm{Zn}(\mathrm{OTf})_{2}{ }^{43}$ and $\mathrm{LaCl}_{3},{ }^{44}$

However, many of these methods suffer from one or more of the drawbacks such as requirement of strong acidic conditions, long reaction times, low yields, tedious work-up procedures, requirement of excess amounts of reagent and use of toxic reagents, catalysts or solvents. Therefore, there is a strong demand for a highly efficient and environmentally benign method.

Lanthanum (III) nitrate have recently attracted much attention in organic transformations due to its high acidity, thermal stability, low toxicity, low cost and good stability, Furthermore, current literature reveals that Lanthanum (III) nitrate has been utilized as an effective catalyst in the synthesis of 4-(3H)-quinazolinones under solvent-free conditions, chiral tetrahydroquinolino pyranose derivatives, chemoselective deprotection of acetonides, chemoselective protection of amines as $\mathrm{N}$ benzyloxycarbonyl derivatives, acetylation of alcohols, phenols and amines with acetic anhydride and synthesis of $\alpha$-amino nitriles. ${ }^{45-50}$

In continuation of our ongoing research to develop novel methodologies in synthetic organic chemistry, ${ }^{51-53}$ we report herein an efficient, low cost and environmentally benign protocol for the synthesis of 2-arylbenzothiazole using Lanthanum (III) nitrate hexahydrate catalyst under mild reaction condition.

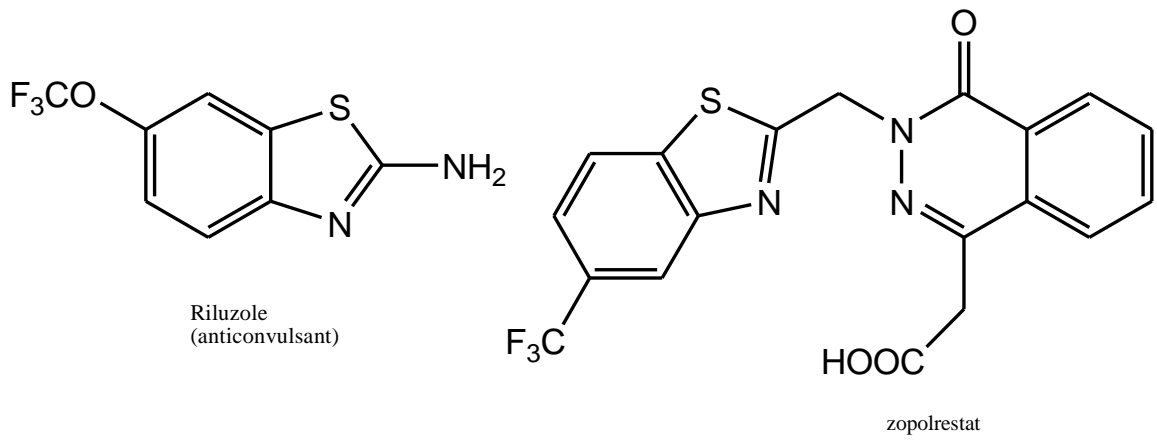

Figure 1. Benzthiazole containing important commercial drugs

\section{Experimental}

\subsection{Materials and Apparatus}

The chemicals and solvents were purchased from commercial suppliers (Merck, S.D. fine and Spectrochem) and they were used without purification prior to use. Melting points were recorded by open tube capillary method and are uncorrected. The progress of the reaction and the purity of the compounds were monitored by thin-layer chromatography (TLC), using analytical silica gel plates (Merck $60 \mathrm{~F} 250$ ). ${ }^{1} \mathrm{H}$ NMR and ${ }^{13} \mathrm{C}$ NMR spectra were recorded on 400 and $100 \mathrm{MHz}$, respectively. NMR spectra were obtained in $\mathrm{CDCl}_{3}$ solutions and are reported as parts per million (ppm) downfield from tetramethylsilane (TMS) as internal standard and the coupling constants $(J)$ are expressed in Hertz $(\mathrm{Hz})$. Mass spectra were recorded on a macro mass spectrometer, applying electrospray ionization (ESI) method. To see the spectra of compounds please see the supporting infotmation (Figure S1-S18). 


\subsection{General Procedure for the Synthesis of 2-Aryl benzothiazole:}

$\mathrm{La}\left(\mathrm{NO}_{3}\right)_{3} 6 \mathrm{H}_{2} \mathrm{O}(15 \mathrm{~mol} \%)$ was added to a stirred solution of the aldehyde $(1.0 \mathrm{mmol})$ and 2aminothiophenol $(1.0 \mathrm{mmol})$ in ethanol $(5 \mathrm{~mL})$ and the mixture was stirred at room temperature for appropriate time (Table 2). The progress of the reaction was monitored by thin layer chromatography (TLC) (Hexane: Ethyl acetate, 8:2). After the reaction was completed, the pure products were isolated by filtration. The solid product was purified by recrystallization from ethanol. Selected spectral data of compounds are given below.

2-(4-Methylphenyl)-benzothiazole (3b) (Table 2 entry $3 b)$ White solid; IR $(\mathrm{KBr}) v\left(\mathrm{~cm}^{-1}\right)$ : 3026, 2811 , $2343,1606,1581,1520,1361,1297,1258,1152,1034,951,867$ and $659 \mathrm{~cm}^{-1} .{ }^{1} \mathrm{H}$ NMR $(400 \mathrm{MHz}$, $\left.\mathrm{CDCl}_{3}\right) \delta$ ppm: $\delta 8.00-8.06(3 \mathrm{H}, \mathrm{m}, \mathrm{Ar}-\mathrm{H}), 7.96(1 \mathrm{H}, \mathrm{d}, J=8.0 \mathrm{~Hz}, \mathrm{Ar}-\mathrm{H}), 7.51(1 \mathrm{H}, \mathrm{t}, J=8.4 \mathrm{~Hz}, \mathrm{Ar}-$ $\mathrm{H}), 7.41(1 \mathrm{H}, \mathrm{t}, J=8.4 \mathrm{~Hz}, \mathrm{Ar}-\mathrm{H}), 7.36(2 \mathrm{H}, \mathrm{d}, J=8.1 \mathrm{~Hz}, \mathrm{Ar}-\mathrm{H}), 2.45\left(3 \mathrm{H}, \mathrm{s},-\mathrm{CH}_{3}\right),{ }^{13} \mathrm{C}$ NMR $\left(100 \mathrm{MHz}, \mathrm{CDCl}_{3}\right) \quad \delta$ ppm: $\delta 168.0,154.2,141.6,135.0,131.0,129.7,127.3,126.2,125.0,122.9$, 121.6 and 21.2; MS: $\mathrm{m} / \mathrm{z} 225.1234[\mathrm{M}+1]^{+}$

2-(4-Methoxyphenyl)-benzothiazole (3c) (Table 2 entry 3c) White solid; IR (KBr) $v\left(\mathrm{~cm}^{-1}\right)$ : 3021, 3048, 2837, 1609, 1590, 1483, 830; ${ }^{1} \mathrm{H}$ NMR $\left(400 \mathrm{MHz}, \mathrm{CDCl}_{3}\right) \delta \mathrm{ppm}: 8.03-8.08(3 \mathrm{H}, \mathrm{m}$, Ar-H) $7.91(1 \mathrm{H}, \mathrm{d}, J=8.0 \mathrm{~Hz}, \mathrm{Ar}-\mathrm{H}), 7.48(1 \mathrm{H}, \mathrm{t},, J=8.0 \mathrm{~Hz}, \mathrm{Ar}-\mathrm{H}), 7.37(1 \mathrm{H}, \mathrm{t}, J=8.0 \mathrm{~Hz}, \mathrm{Ar}-\mathrm{H}), 7.02(2 \mathrm{H}$, $\mathrm{d}, J=8.4 \mathrm{~Hz}, \mathrm{Ar}-\mathrm{H}), 3.89\left(3 \mathrm{H}, \mathrm{s}, \mathrm{OCH}_{3}\right) ;{ }^{13} \mathrm{C} \mathrm{NMR}\left(100 \mathrm{MHz}, \mathrm{CDCl}_{3}\right) \delta \mathrm{ppm}:, 167.6,162.0,154.3$, 134.9, 129.0, 126.3, 126.1, 124.7, 122.7, 121.5, 114.3, 55.4; MS: $\mathrm{m} / \mathrm{z} 242.0649[\mathrm{M}+1]^{+}$

2-(4-Chlorophenyl)-benzothiazole (3d) (Table 2 entry 3d) Yellow solid; IR (KBr) $v\left(\mathrm{~cm}^{-1}\right)$ : 3051, 2326, 1600, 1434, 1372, 1313, 1168, 1064, 945, 821; ${ }^{1} \mathrm{H}$ NMR $\left(400 \mathrm{MHz}, \mathrm{CDCl}_{3}\right) \delta \mathrm{ppm:} 8.06(1 \mathrm{H}$, $\mathrm{d}, J=8.2 \mathrm{~Hz}, \mathrm{Ar}-\mathrm{H}), 8.01(2 \mathrm{H}, \mathrm{d}, J=8.5 \mathrm{~Hz}, \mathrm{Ar}-\mathrm{H}), 7.89(1 \mathrm{H}, \mathrm{d}, J=8.0 \mathrm{~Hz}, \mathrm{Ar}-\mathrm{H}), 7.47(3 \mathrm{H}, \mathrm{m}, \mathrm{Ar}-$ $\mathrm{H}), 7.40-7.36(1 \mathrm{H}, \mathrm{m}, \mathrm{Ar}-\mathrm{H}) ;{ }^{13} \mathrm{C}$ NMR $\left(100 \mathrm{MHz}, \mathrm{CDCl}_{3}\right) \quad \delta \mathrm{ppm}: 166.5,154.0,137.0,135.0,132.1$, 129.2, 128.7, 126.4, 125.4, 123.3, 121.6; MS: $m / z$ 246.0143 [M + 1] ${ }^{+}$

2-(4-N,N-Dimethylphenyl)-benzothiazole (3g) (Table 2 entry $3 g$ ) Yellow solid; IR $(\mathrm{KBr}) \quad v\left(\mathrm{~cm}^{-1}\right)$ : 3052, 1600, 1435, 1372, 1313, 1168, 1037, 946, 821; ${ }^{1} \mathrm{H}$ NMR $\left(400 \mathrm{MHz}, \mathrm{CDCl}_{3}\right) \delta$ ppm: $7.86(2 \mathrm{H}$, $\mathrm{d}, J=8.5 \mathrm{~Hz}) ; 7.62(1 \mathrm{H}, \mathrm{d}, J=7.8 \mathrm{~Hz}) ; 7.16(1 \mathrm{H}, \mathrm{t}, J=7.7 \mathrm{~Hz}) ; 7.08(1 \mathrm{H}, \mathrm{t}, J=7.6 \mathrm{~Hz}) ; 7.02(1 \mathrm{H}, \mathrm{d}$, $J=7.6 \mathrm{~Hz}) ; 6.75(2 \mathrm{H}, \mathrm{d}, J=8.9 \mathrm{~Hz}) ; 3.07(6 \mathrm{H}, \mathrm{s}) ;{ }^{13} \mathrm{C} \mathrm{NMR}\left(100 \mathrm{MHz}, \mathrm{CDCl}_{3}\right) \quad \delta \mathrm{ppm}: 159.59,152.7$, 149.7, 131.8, 130.8, 128.8, 126.5, 125.8, 125.5, 124.4, 117.1, 111.5, 40.1; MS: $m / z$ 254.09, 157.9, $131.9,114.0,102.1,86.2,72.4$

2-(4-Bromophenyl)-benzothiazole $(3 \boldsymbol{k})$ (Table 2 entry $3 k$ ) White solid; IR $(\mathrm{KBr}) \vee\left(\mathrm{cm}^{-1}\right)$ : 3000, 14001600. ${ }^{1} \mathrm{H}$ NMR $\left(400 \mathrm{MHz}, \mathrm{CDCl}_{3}\right): \delta \mathrm{ppm}: 8.11(1 \mathrm{H}, \mathrm{d}, J=8.0 \mathrm{~Hz}, \mathrm{Ar}-\mathrm{H}), 8.0(2 \mathrm{H}, \mathrm{d}, J=8.5 \mathrm{~Hz}, \mathrm{Ar}-$ $\mathrm{H}), 7.94(1 \mathrm{H}, \mathrm{d}, J=8.0 \mathrm{~Hz}, \mathrm{Ar}-\mathrm{H}), 7.67(2 \mathrm{H}, \mathrm{d}, J=8.5 \mathrm{~Hz}, \mathrm{Ar}-\mathrm{H}), 7.55(1 \mathrm{H}, \mathrm{t}, J=8.0 \mathrm{~Hz}, \mathrm{Ar}-\mathrm{H}), 7.44$ $(1 \mathrm{H}, \mathrm{t}, J=8.0 \mathrm{~Hz}, \mathrm{Ar}-\mathrm{H}) .{ }^{13} \mathrm{C}$ NMR $\left(100 \mathrm{MHz}, \mathrm{CDCl}_{3}\right): \delta \mathrm{ppm}: 167.1,154.5,135.4,133.0,132.6$, 129.3, 126.9, 125.8, $125.8,123.7,122.0$; MS: $m / z 289.9638[\mathrm{M}+1]^{+}$

2-(3-Nitrophenyl)-benzothiazole (3m) (Table 2 entry $3 m$ ) Yellow solid; IR $(\mathrm{KBr}) \quad v\left(\mathrm{~cm}^{-1}\right)$ : 3080, 3035, 1612, 1580; ${ }^{1} \mathrm{H}$ NMR (400 MHz, $\left.\mathrm{CDCl}_{3}\right): \delta \mathrm{ppm}: 8.95(1 \mathrm{H}, \mathrm{d} J=8.0 \mathrm{~Hz}), 8.45(1 \mathrm{H}, \mathrm{d}, \mathrm{J}=8.0$ $\mathrm{Hz}, 1 \mathrm{H}), 8.36(1 \mathrm{H}, \mathrm{d}, J=8.0 \mathrm{~Hz}), 8.15(1 \mathrm{H}, \mathrm{d}, J=8.0 \mathrm{~Hz}), 7.98(1 \mathrm{H}, \mathrm{d}, J=8.0 \mathrm{~Hz}), 7.73(1 \mathrm{H}, \mathrm{t}, J=7.9$ $\mathrm{Hz}), 7.59(1 \mathrm{H}, J=7.5 \mathrm{~Hz}), 7.49(1 \mathrm{H}, J=7.6 \mathrm{~Hz}) ;{ }^{13} \mathrm{C} \mathrm{NMR}\left(100 \mathrm{MHz}, \mathrm{CDCl}_{3}\right): \delta \mathrm{ppm}: 164.9,153.9$, $148.7,135.3,135.1,133.0,130.1,126.8,126.0,125.2,123.7,122.3,121.8, \mathrm{MS}: \mathrm{m} / z .257 .0379[\mathrm{M}+$ $1]^{+}$

\section{Results and Discussion}

To explore the use of Lanthanum (III) nitrate hexahydrate as a catalyst, a reaction of benzaldehyde $\mathbf{2}$ and 2- aminothiophenol $\mathbf{1}$ was conducted as a standard model reaction for the preparation of 2-arylbenzothiazoles (3a-3n) (Scheme 1). The reaction in the absence of catalyst did 
not give any desired product. To determine the exact amount of the catalyst, we investigated the model reaction using different concentrations of Lanthanum (III) nitrate hexahydrate (Table 1). During this study, we observed that $15 \mathrm{~mol} \%$ Lanthanum (III) nitrate hexahydrate was proved to be an efficient catalyst to conduct the reaction smoothly. With these optimized reaction conditions, effect of different solvents such as methanol, dichloromethane, acetonitrile, THF, ethanol, aqueous ethanol and water was investigated (entries 1-7, Table 1). Among the tested solvents, ethanol was found to be superior over the other tested solvents in terms of both yield and reaction time (Table 1 Entry 10) for this transformation.

Table 1. Effect of solvent and catalyst evaluation in synthesis of 2-phenylbenzothiazole at room temperature $^{\mathrm{a}}$

\begin{tabular}{ccccc}
\hline Entry & Solvent & Catalyst (mol \%) & Time (min.) & Yield $^{\mathbf{b}}(\%)$ \\
\hline 1 & $\mathrm{THF}$ & 10 & 45 & 62 \\
2 & $\mathrm{CH}_{3} \mathrm{CN}$ & 10 & 45 & 72 \\
3 & $\mathrm{CH}_{3} \mathrm{OH}$ & 10 & 45 & 82 \\
4 & $\mathrm{CH}_{2} \mathrm{Cl}_{2}$ & 10 & 45 & 56 \\
5 & $\mathrm{H}_{2} \mathrm{O}$ & 10 & 45 & 60 \\
6 & $\mathrm{EtOH}: \mathrm{H}_{2} \mathrm{O}$ & 10 & 45 & 68 \\
7 & $\mathrm{EtOH}$ & 5 & 57 & 75 \\
9 & $\mathrm{EtOH}$ & 7 & 50 & 80 \\
10 & EtOH & 10 & 45 & 88 \\
11 & EtOH & 15 & 30 & 94
\end{tabular}

${ }^{a}$ Reaction conditions: benzaldehyde $(1 \mathrm{mmol})$, 2-aminothiophenol (1 mmol), Lanthanum (III) nitrate hexahydrate $(15 \mathrm{~mol} \%)$ in ethanol $(5 \mathrm{~mL})$ at room temperature. ${ }^{\mathrm{b}}$ Isolated yield.

Encouraged by this result, in order to build the generality of the reaction, various aromatic aldehydes, posessing electron-donating and electron-withdrawing groups were converted to 2arylbenzothiazole derivatives in good to excellent yields. All the results are summarized in Table 2.

In order to understand the efficiency and greenness of the method, we compared our results on the synthesis of 2-phenyl benzothiazole with the well-known data from the literature (Table 3). As shown in the Table 3, many of the previously reported methodologies suffer from one or more disadvantages such as requirement of excess amount of catalyst, high temperature, ultrasound irradiation, prolonged reaction time, and use of volatile and toxic organic solvents. Thus, the present method avoids the disadvantages of the previously reported methodologies.

The mechanism for 2-aryl benzothiazole formation is proposed in scheme 2. Lanthanum (III) nitrate hexahydrate appears to play a more efficient catalytic role due to strong oxophilicity. Initially, aldehyde molecules co-ordinate through their carbonyl oxygen atoms to the lanthanum ion and facilitate the nucleophilic attack. The reaction between an aldehyde and thiol leads to the formation of sulfonium ion intermediate. Then, intramolecular attack by the second group on $\mathrm{C}=\mathrm{S}$ double bond followed by air oxidation gives the final product. 

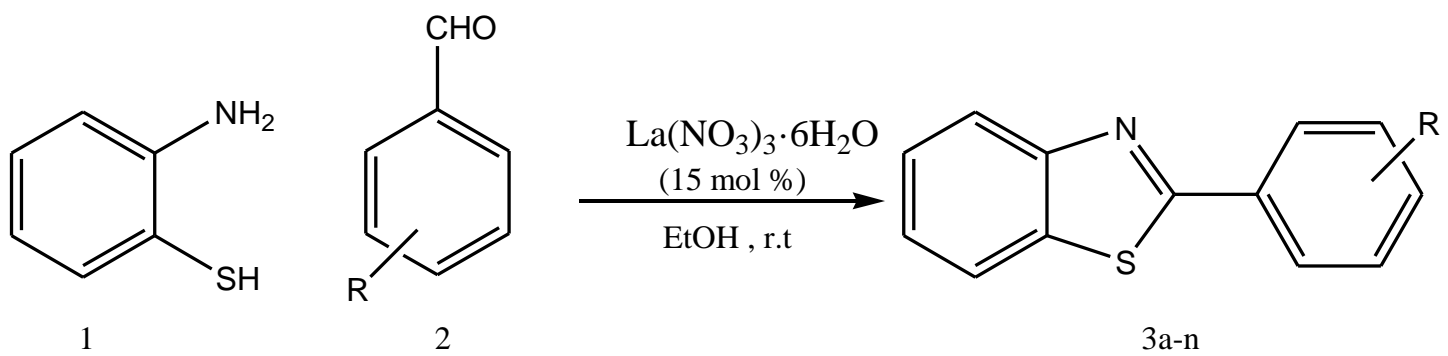

Scheme 1. Synthesis of 2-arylbenzothiazole

Table 2. Synthesis of 2-arylbenzothiazole in the presence of Lanthanum (III) nitrate hexahydrate ${ }^{\mathrm{a}}$

\begin{tabular}{|c|c|c|c|c|c|}
\hline Entry & Aldehydes & Products & $\begin{array}{l}\text { Time } \\
(\text { min) }\end{array}$ & $\begin{array}{c}\text { Yield }^{b} \\
(\%)\end{array}$ & $\begin{array}{c}\text { M.P } \\
{ }^{\circ} \mathrm{C} \text { [Ref.] }\end{array}$ \\
\hline & & & 30 & 94 & $112-114$ [14] \\
\hline & & & 35 & 96 & $80-82$ [27] \\
\hline & & & 35 & 94 & $125-126$ [14] \\
\hline $3 d$ & & & 30 & 96 & 114-116 [27] \\
\hline & & & 32 & 96 & $224-226[14]$ \\
\hline & & & 40 & 92 & $228-230$ [27] \\
\hline
\end{tabular}


Table 2 Continued.

$3 g$<smiles>CCN(C)c1ccc(C=O)cc1</smiles>

3h<smiles>O=Cc1ccccc1Cl</smiles>

3i<smiles>O=Cc1ccccc1[N+](=O)[O-]</smiles>

3j<smiles>O=Cc1ccccc1O</smiles>

3k<smiles>O=Cc1ccc(Br)cc1</smiles>

3I<smiles>O=Cc1ccc(Cl)cc1Cl</smiles>

$3 m$<smiles>O=Cc1cccc([N+](=O)[O-])c1</smiles>

3n

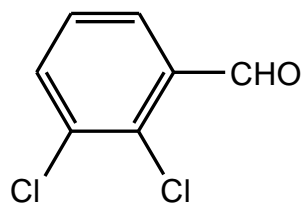<smiles>CN(C)c1ccc(-c2nc3ccccc3s2)cc1</smiles>

172-174 [27]

35

90

74-76 [14]<smiles>Clc1ccccc1-c1nc2ccccc2s1</smiles><smiles>O=[N+]([O-])c1ccccc1-c1nc2ccccc2s1</smiles><smiles>Oc1ccccc1-c1nc2ccccc2s1</smiles><smiles>Brc1ccc(-c2nc3ccccc3s2)cc1</smiles><smiles>Clc1ccc(-c2nc3ccccc3s2)c(Cl)c1</smiles><smiles>O=[N+]([O-])c1cccc(-c2nc3ccccc3s2)c1</smiles> 
Table 3. Comparison of the activity of various catalysts for the synthesis of 2-arylbenzothiazole from the condensation of benzaldehyde and 2-aminothiophenol

\begin{tabular}{|c|c|c|c|c|c|}
\hline Entry & Catalyst & $\begin{array}{c}\text { Amount of } \\
\text { catalyst }\end{array}$ & Condition & Time & Yield (\%) [Ref.] \\
\hline 1 & $\mathrm{Co}\left(\mathrm{NO}_{3}\right)_{2} 6 \mathrm{H}_{2} \mathrm{O}$ & $2 \mathrm{mmol}$ & $\mathrm{DMF} / 80^{\circ} \mathrm{C}$ & $15 \min$ & 93 [39] \\
\hline 2 & $\mathrm{Ni}\left(\mathrm{NO}_{3}\right)_{2} 6 \mathrm{H}_{2} \mathrm{O}$ & $2 \mathrm{mmol}$ & $\mathrm{DMF} / 80^{\circ} \mathrm{C}$ & $90 \min$ & 85 [39] \\
\hline 3 & $\mathrm{Fe}\left(\mathrm{NO}_{3}\right)_{3}: 9 \mathrm{H}_{2} \mathrm{O}$ & $5 \mathrm{~mol} \%$ & $\begin{array}{c}\mathrm{P}(\mathrm{tBu})_{3:} \mathrm{HBF}_{4} \\
\text { DMSO: } \mathrm{H}_{2} \mathrm{O}, 120^{\circ} \mathrm{C}\end{array}$ & $24 \mathrm{~h}$ & 89 [40] \\
\hline 4 & $\mathrm{Bi}\left(\mathrm{NO}_{3}\right)_{3}$ & $0.15 \mathrm{mmol}$ & $\begin{array}{l}\text { aq. } 30 \% \mathrm{H}_{2} \mathrm{O}_{2} \text { in } \\
\text { ethanol, Reflux }\end{array}$ & $20 \min$ & 90 [41] \\
\hline 5 & $\mathrm{CuSO}_{4}$ & $10 \mathrm{~mol} \mathrm{\%}$ & Glycerol / )))) & $70 \min$ & $91 \quad[42]$ \\
\hline 6 & $\operatorname{Sm}(\mathrm{OTf})_{3}$ & $10 \mathrm{~mol} \%$ & $\begin{array}{c}\mathrm{EtOH}: \mathrm{H}_{2} \mathrm{O} \\
50-60{ }^{\circ} \mathrm{C}\end{array}$ & $2-5 \mathrm{~h}$ & 89 [31-32] \\
\hline 7 & $\mathrm{Zn}(\mathrm{OTf})_{2}$ & $10 \mathrm{~mol} \mathrm{\%}$ & Ethanol,Reflux & $5 \mathrm{~h}$ & 92 [43] \\
\hline 8 & $\mathrm{Fe}_{3} \mathrm{O}_{4} @ \mathrm{SiO}_{2} /$ Collagen & $50 \mathrm{mg}$ & EtOH, Reflux & $1.5 \mathrm{~h}$ & $70 \quad[36]$ \\
\hline 9 & $\mathrm{VOSO}_{4}$ & $3 \mathrm{~mol} \%$ & $\mathrm{EtOH}$, r.t & $60 \min$ & 92 [38] \\
\hline 10 & $\mathrm{BF}_{3} / \mathrm{SiO}_{2}$ & $\underset{\mathrm{BF}_{3}}{0.05 \mathrm{~g}, 25 \mathrm{~mol} \%}$ & $\mathrm{EtOH}, \mathrm{r.t}$ & $30 \mathrm{~min}$ & 85 [33] \\
\hline \multirow[t]{2}{*}{12} & $\mathrm{La}\left(\mathrm{NO}_{3}\right)_{3} \cdot 6 \mathrm{H}_{2} \mathrm{O}$ & $15 \mathrm{~mol} \%$ & $\mathrm{EtOH}, \mathrm{r.t}$ & $30 \mathrm{~min}$ & 94 \\
\hline & & & & & Present work \\
\hline
\end{tabular}

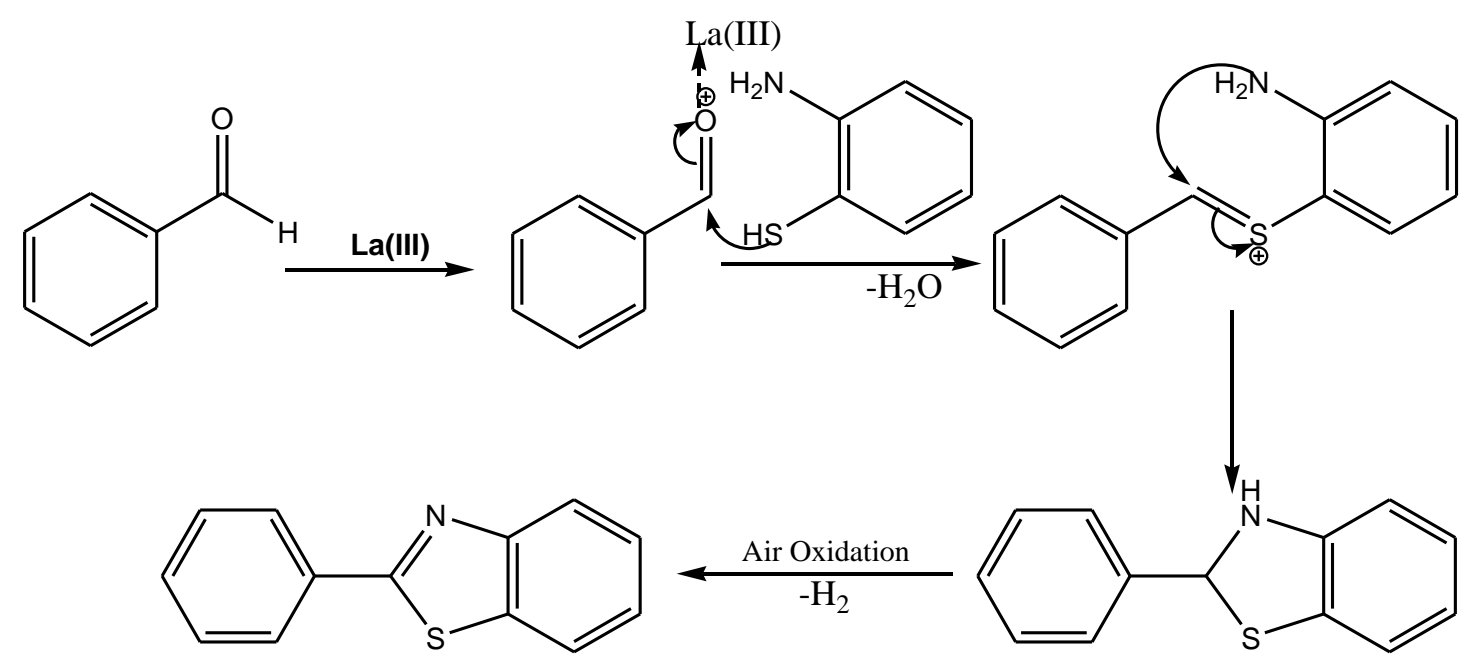

Scheme 2. A plausible mechanism for synthesis of 2-arylbenzothiazoles in the presence of Lanthanum (III) nitrate hexahydrate 


\section{Conclusion}

In summary, we have developed a facile, efficient and green method for the synthesis of 2substituted benzothiazoles through condensation of aromatic aldehydes with 2-aminothiophenol in the presence of Lanthanum (III) nitrate hexahydrate under mild reaction conditions. Compare with the previously reported methodologies, the present protocol features simple work-up, environmentally benign, high yields and use of catalytic amount of a cheap catalyst.

\section{Acknowledgements}

Authors are pleased to acknowledge financial support received from Department of Science and Technology (SERB/F/4992/2013-14 dated 30/01/2013), New Delhi. Authors are also thankful to Principal, Kalikadevi (ACS) College, Shirur (Ka.) Dist. Beed for providing necessary laboratory facilities to carry out this work.

\section{Supporting Information}

Supporting Information accompanies this paper on http://www.acgpubs.org/OC

\section{ORCID}

Kabeer A. Shaikh: 0000-0003-0814-3658

Uddhav N. Chaudhar: 0000-0002-7593-978X

\section{References}

[1] Song, X.; Vig, B. S.; Lorenzi, P. L.; Drach, J. C.; Townsend, L. B.; Amidon, G. L. Amino Acid ester prodrugs of the antiviral agent 2-bromo-5,6-dichloro-1-( $\beta$-D-ribofuranosyl) benzimidazole as potential substrates of hPEPT1 transporter. J. Med. Chem. 2005, 48, 1274-1277.

[2] Evans, D. A.; Sacks, C. E.; Kleschick, W. A.; Taber, T. R. Polyether antibiotics synthesis. Total synthesis and absolute configuration of the ionophore A-23187. J. Am. Chem. Soc. 1979, 101, 6789-6791.

[3] Yamato, M. Study on the development of biological-active compounds after the model of natural products. J. Pharm. Soc. Jpn. 1992, 112, 81-99.

[4] Yildiz-Oren, I.; Yalcin, I.; Aki-Sener, E.;Ucarturk, N. Synthesis and structure-activity relationships of new antimicrobial active multisubstituted benzazole derivatives. Eur. J. Med. Chem. 2004, 39, 291-298.

[5] Benazzouz, A.; Boraud, T.; Dub'edat, P.; Boireau, A.; Stutzmann, J.M.; Gross, C. Prevents MPTP-induced parkinsonism in the rhesus monkey: A pilot study. Eur. J. Pharmacol. 1995, 284, 299-307.

[6] Kumar, D.; Jacob, M. R.; Reynolds, M. B.; Kerwin, S.M. Synthesis and evaluation of anticancer benzoxazoles and benzimidazoles related to UK-1. Bioorg. Med. Chem. 2002, 10, 3997-4004.

[7] Mortimer, C. G.; Wells, G.; Crochard, J.P.; Stone, E. L.; Bradshaw, T. D.; Stevens, M. F. G.; Westwell, A. D. Antitumor benzothiazoles. 26. 2-(3,4-Dimethoxyphenyl)-5-fluorobenzothiazole (GW 610, NSC 721648), a simple fluorinated 2-Arylbenzothiazole, shows potent and selective inhibitory activity against lung, colon, and breast cancer cell linesi. J. Med. Chem. 2006, 49, 179-185.

[8] Mylari, B. L.; Larson, E. R.; Beyer, T. A.; Zembrowski, W. J.; Aldinger, C. E.; Dee, M. F.; Siegel, T. W.; Singleton, D. H. Novel, potent aldose reductase inhibitors: 3,4-dihydro-4-oxo-3-[[5-(trifluoromethyl)-2benzothiazolyl]methyl]-1-phthalazineacetic acid (zopolrestat) and congeners. J. Med. Chem. 1991, 34, 108122.

[9] Mylari, B. L.; Beyer, T. A.; Scott, P. J.; Aldinger, C. E.; Dee, M. F.; Siegel, T. W.; Zembrowski, W. J. Potent, orally active aldose reductase inhibitors related to zopolrestat: Surrogates for benzothiazole side chain. J. Med. Chem. 1992, 35, 457-465.

[10] Song, J.H.; Huang, C.S.; Nagata, K.; Yeh, J. Z.; Narahashi, T. Differential Action of Riluzole on Tetrodotoxin-Sensitive and Tetrodotoxin-Resistant Sodium Channels, J. Pharmacol. Exp.Ther. 1997, 282, 707-714.

[11] Kodomari, M.; Noguchi, T.; Aoyama, T. Solvent-Free synthesis of 1,5-Benzothiazepines and Benzodiazepines on inorganic supports. Synth. Commun. 2004, 34, 1783-1790. 
[12] Patil, A. V.; Bandgar, B. P.; Lee, S. H. Silica Supported Fluoroboric Acid: An Efficient and Reusable Heterogeneous Catalyst for Facile Synthesis of 2-Aliphatic Benzothiazoles, Benzoxazoles, Benzimidazoles and Imidazo[4,5-b]pyridines. Bull. Korean Chem. Soc. 2010, 31, 1719-1722.

[13] Pratap, U. R.; Mali, J. R.; Jawale, D.V.; Mane, R. A. Bakers' yeast catalyzed synthesis of benzothiazoles in an organic medium. Tetrahedron Lett. 2009, 50, 1352-1354.

[14] Al-Qalaf, F.; Mekheimer, R. A.; Sadek, K. U. Cerium (IV) ammonium Nitrate (CAN) catalyzed one-pot synthesis of 2-arylbenzothiazoles. Molecules, 2008, 13, 2908-2914.

[15] Ding, Q.; He, X.; Wu, J. Synthesis of 2-aminobenzothiazole via copper (I)-catalyzed Tandem Reaction of 2-iodobenzenamine with isothiocyanate. J. Comb. Chem. 2009, 11, 587-591.

[16] Kumar, A.; Maurya, R. A.; Saxena, D. Diversity-oriented synthesis of benzimidazole, benzoxazole, benzothiazole and quinazolin-4(3H)-one libraries via potassium persulfate- $\mathrm{CuSO}_{4}$-mediated oxidative coupling reactions of aldehydes in aqueous micelles. Mol. Divers. 2010, 14, 331-341.

[17] Yang, Z.; Chen, Xi.; Wang, S.; Liu, J.; Xie, Kai; Wang, A.; Tan, Ze. Synthesis of 2-aryl benzothiazoles via $\mathrm{K}_{2} \mathrm{~S}_{2} \mathrm{O}_{8}$ mediated oxidative condensation of benzothiazoles with aryl aldehydes. J. Org. Chem. 2012, 77, 7086-7091.

[18] Bahrami, K.; Khodaei, M. M.; Nejati, A. Synthesis of 1,2-disubstituted benzimidazoles, 2-substituted benzimidazoles and 2-substituted benzothiazoles in SDS micelles. Green Chem. 2010, 12, 1237-1241.

[19] Mali, J. R.; Jawale, D.V.; Londhe, B. S.; Mane, R. A. An efficient green protocol for the synthesis of 2-aryl substituted benzothiazoles. Green Chem. Lett. Rev. 2010, 3, 209-212

[20] Goswami, P.; Das, B. Organocatalyzed one-Pot synthesis of substituted 1,5-benzodiazepine and benzimidazole derivatives. Synth. Commun. 2010, 40, 1685-1693.

[21] Azizi, N.; Amiri, A. K.; Baghi, R.; Bolourtchian, M.; Hashemi; M. M. PTSA catalyzed simple and green synthesis of benzothiazole derivatives in water. Monatsh. Chem. 2009, 140, 1471-1473.

[22] Yang, X.L.; Xu, Chun-Mei; Lin, S.M.; Chen, Jiu-Xi; Ding, J.C.; Wu, H.Y.; Su, Wei-K. Eco-Friendly synthesis of 2-substituted benzothiazoles catalyzed by cetyltrimethyl ammonium bromide (CTAB) in water. J. Braz. Chem. Soc. 2010, 21, 37-42.

[23] Azarifar, D.; Maleki, B.; Setayeshnazar, M. A Simple, microwave-assisted, and solvent-free synthesis of 2arylbenzothiazoles by acetic acid-promoted condensation of aldehydes with 2-aminothiophenol in air. Phosphor., Sulfur Sil. 2009, 184, 2097-2102.

[24] Patil, D. R.; Salunkhe, S.M.; Sambavekar, P. P.; Deshmukh, M. B.; Kolekar, G. B.; Anbhule, P. V. Silica sulfuric acid: Recyclable and efficient catalyst for the 2-aryl benzothiazoles. Der Pharm. Chem. 2011, 3, 189-193.

[25] Praveen, C.; Nandakumar, A.; Dheenkumar, P.; Muralidharan, D.; Perumal, P. T. Microwave-assisted onepot synthesis of benzothiazole and benzoxazole libraries as analgesic agents. J. Chem. Sci. 2012,124, 609624.

[26] Ziarani, G. M.; Badiei, A.; Nahad, M. S.; Hassanzadeh, M. Application of SBA-Pr-SO ${ }_{3} \mathrm{H}$ in the synthesis of benzoxazole derivatives. Eur. J. Chem. 2012, 3, 433-436.

[27] Rostami, A.; Yari, A. Sulfamic acid as a recyclable and green catalyst for rapid and highly efficient synthesis of 2-arylbenzothiazoles in water at room temperature. J. Iran. Chem. Soc. 2012, 9,489-493.

[28] Sharghi, H.; Aberi, M.; Doroodmand, M. M. One-pot synthesis of 2-arylbenzimidazole, 2arylbenzothiazole and 2-arylbenzoxazole derivatives using vanadium(IV)-salen complex as homogeneous catalyst and vanadium(IV)-salen complex nanoparticles immobilized onto silica as a heterogeneous nanocatalyst. J. Iran. Chem. Soc. 2012, 9,189-204.

[29] Chen, G. F.; Jia, H. M.; Zhang, L. Y.; Chen, B. H.; Li, Ji-T. An efficient synthesis of 2-substituted benzothiazoles in the presence of $\mathrm{FeCl}_{3}$ /Montmorillonite $\mathrm{K}-10$ under ultrasound irradiation. Ultrason. Sonochem. 2013, 20, 627-632.

[30] Fortenberry, C.; Nammalwar, B.; Bunce, R. A. Ammonium chloride-catalyzed synthesis of benzo-fused heterocycles from $o$-Substituted anilines and orthoesters. Org. Preps Proc.. Int. 2013, 45, 57-65.

[31] Gorepatil, P. B.; Mane, Y. D.; Gorepatil, A. B.; Gaikwad, M. V.; Ingle, V. S. Samarium (III) triflate: A new catalyst for facile synthesis of benzothiazoles and benzoxazoles from carboxylic acids in aqueous media. Res. Chem. Intermed. 2014, 41, 8355-8362.

[32] Gorepatil, P. B.; Mane, Y.D.; Ingle, V.S. Samarium (III) triflate is a efficient and reusable catalyst for facile synthesis of benzothiazoles and benzoxazoles in aqueous media. Synlett, 2013, 24, 2241-2244.

[33] Naeimi, H.; Heidarnezhad, A.; Synthesis of 2-arylbenzothiazoles using nano $\mathrm{BF}_{3} / \mathrm{SiO}_{2}$ as a reusable and efficient heterogeneous catalyst under mild conditions. J. Sulfur Chem. 2014, 35, 493-501. 
[34] Gill, C.H.; Nikam, M.D.; Mahajan, P.S.; Chate, A.V.; Dabhade, S.K.; Badadhe, P.V. Lithium bromide catalyzed efficient and convenient synthesis of 2-arylbenzothiazole derivatives. Res. Chem. Intermed. 2015, 41, 7509-7516.

[35] Soleimani, E.; Khodaei, M. M.; Yazdani, H.; Saei, P.; Reza, J. Z. Synthesis of 2-substituted benzimidazoles and benzothiazoles using $\mathrm{Ag}_{2} \mathrm{CO}_{3} /$ Celite as an efficient solid catalyst. J. Iran. Chem. Soc. 2015, 12,1281-1285.

[36] Ghafuri, H.; Esmaili, E.; Talebi, M.. $\mathrm{Fe}_{3} \mathrm{O}_{4} \cdot \mathrm{SiO}_{2} /$ collagen: An efficient magnetic nanocatalyst for the synthesis of benzimidazole and benzothiazole derivatives. C. R. Chimie, 2016, 19, 942-950.

[37] Schoultz, X.; Gerber, T.I.A.; Hosten, E.C. Rhenium (I) complexes with benzothiazole-thiourea derivatives. Polyhedron, 2016, 113, 55-60.

[38] Singh Digwal, C.; Yadav, U.; Sakla, A. P.; Sri Ramya, P. V.; Aaghaz, S.; Kamal, A. VOSO 4 catalyzed highly efficient synthesis of benzimidazoles, benzothiazoles and quinoxalines. Tetrahedron Lett. 2016, 57, $4012-4016$

[39] Chandrachood, P. S.; Garud, D. R.; Gadakari, T. V.; Torane, R. C.; Deshpande, N. R.; Kashalkar, R. V. A Cobalt Nitrate/Hydrogen oeroxide system as an efficient reagent for the synthesis of 2-aryl benzimidazoles and benzothiazoles. Acta Chim. Slov. 2011, 58, 367-371.

[40] Khemnar, A. B.; Bhanage, B. M. Iron catalyzed efficient synthesis of 2-arylbenzothiazoles from benzothiazole and olefins using environmentally benign molecular oxygen as oxidant. $R S C A d v .2014,4$, 8939-8942.

[41] Ghashang, M. Bismuth nitrate as an efficient catalyst for the preparation of 2-arylbenzothiazole derivatives. Res. Chem. Intermed. 2014, 40, 1669-1674.

[42] Karimi, R. R.; Talebizadeh, A.; Zirtol, L. A. Rapid and green synthesis of some benzothiazole-, benzimidazole- and benzoxazole-2-thiol derivatives using copper sulfate in aqueous media. Org. Chem. Res. 2016, 2, 64-69.

[43] Srinivasulu, R.; Kumar, K. R.; Satyanarayana, P.V.V.; Babu, B. H. Synthesis of benzothiazole derivatives catalyzed by Zinc triflate J. Applied Chem. 2014, 2 ,5-9.

[44] Venkateswarlu, Y.; Kumar, S. R.; Leelavathi, P. Facile and efficient one-pot synthesis of benzimidazoles using lanthanum chloride. Org.Med.Chem. Lett. 2013 3, 7-12.

[45] Narasimhulu, M.; Mahesh, K. C.; Reddy, T. S.; Rajesh, K.;Venkateswarlu, Y. Lanthanum(III) nitrate hexahydrate or p-toluenesulfonic acid catalyzed one-pot synthesis of 4(3H)-quinazolinones under solventfree conditions. Tetrahedron Lett. 2006, 47, 4381-4383.

[46] Narasimhulu, M.; Reddy, S. M.; Rajesh, K.; Suryakiran, N.; Ramesh, D.; Venkateswarlu, Y. A mild and efficient synthesis of chiral tetrahydroquinolino pyranose derivatives catalyzed by lanthanum(iii) nitrate hexahydrate. Heteroatom Chem. 2008, 19, 429-433.

[47] Reddy, S. M.; Reddy, Y. V.; Venkateswarlu, Y. A mild and efficient method for the chemoselective deprotection of acetonides with lanthanum(III) nitrate hexahydrate. Tetrahedron Lett. 2005, 46, 7439-7441.

[48] Mahesh, K. C.; Narasimhulu, M.; Reddy, T. S.; Suryakiran, N.; Venkateswarlu, Y. A mild and efficient chemoselective protection of amines as $N$-benzyloxycarbonyl derivatives in the presence of $\mathrm{La}\left(\mathrm{NO}_{3}\right)_{3} \cdot 6 \mathrm{H}_{2} \mathrm{O}$ under solvent-free conditions. Tetrahedron Lett. 2007, 48, 55-59.

[49] Reddy, T. S.; Narasimhulu, M.; Suryakiran, N.; Mahesh, K. C.; Ashalatha, K.; Venkateswarlu, Y. A mild and efficient acetylation of alcohols, phenols and amines with acetic anhydride using $\mathrm{La}\left(\mathrm{NO}_{3}\right)_{3} \cdot 6 \mathrm{H}_{2} \mathrm{O}$ as a catalyst under solvent-free conditions. Tetrahedron Lett. 2006, 47, 6825-6829.

[50] Narasimhulu, M.; Reddy, T. S.; Mahesh, K. C.; Reddy, S. M.; Reddy, A. V.; Venkateswarlu, Y. Lanthanum (III) nitrate hexahydrate or gadolinium(III) chloride hexahydrate catalyzed one-pot synthesis of $\alpha$-amino nitriles. J. Mol. Catalysis A: Chemical. 2007, 264, 288-292.

[51] Shaikh, K.A.; Patil, V.A.; Arshia, P. An efficient and convenient synthesis of imidazolines and benzimidazoles via oxidation of carbon-nitrogen bond in water media. Chin. J. Chem. 2012, 30, 924-928.

[52] Shaikh, K. A.; Patil, V.A. An efficient solvent-free synthesis of imidazolines and benzimidazoles using $\mathrm{K}_{4}$ $\left[\mathrm{Fe}(\mathrm{CN})_{6}\right]$ catalysis. Org. Commun. 2012, 5, 12-17.

[53] Shaikh, K. A.; Chaudhar, U. N.; Ningdale, V. B.; A facile and rapid access towards the synthesis of 2-aryl benzothiazoles using succinimide- $N$-sulphonic acid: A Reusable catalyst. Can. Chem. Trans. 2016, 4, 133142.

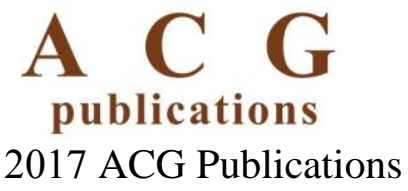

